\title{
Respiratory bronchiolitis-interstitial lung disease
}

\author{
Alicja Sieminska ${ }^{*}$ and Krzysztof Kuziemski
}

\begin{abstract}
Respiratory bronchiolitis-associated interstitial lung disease (RB-ILD) is a rare, mild inflammatory pulmonary disorder that occurs almost exclusively in current or former heavy smokers, usually between the third and sixth decades, most likely with no gender predilection. The onset is usually insidious with exertional dyspnea and persistent cough, which may be non-productive, developing over a course of weeks or months. RB-ILD may also be diagnosed in asymptomatic patients with functional impairment and chest radiograph or high-resolution computed tomography (HRCT) abnormalities. Histologically, RB-ILD is characterized by the accumulation of yellow-brown pigmented macrophages within the lumens of respiratory bronchioles and alveolar ducts, associated with a patchy submucosal and peribronchiolar chronic inflammation. Common findings also include mild bronchiolar and peribronchiolar alveolar fibrosis that expands contiguous alveolar septa and leads to architectural distortion as well as centrilobular emphysema. Chest radiographs in patients with RB-ILD typically show fine reticulonodular interstitial opacities, while on HRCT central and peripheral bronchial wall thickening, centrilobular nodules, and ground-glass opacities associated with upper lobe centrilobular emphysema are most frequently reported. Pulmonary function testing may be normal but usually demonstrates mixed, predominantly obstructive abnormalities, often combined with hyperinflation and usually associated with a mild to moderate reduction in carbon monoxide diffusion capacity (DLCO). The course of RB-ILD is heterogeneous. Some patients respond favorably to corticosteroids and/or smoking cessation, but often there is no functional improvement and the disease progresses despite smoking cessation and treatment.
\end{abstract}

Keywords: Respiratory bronchiolitis-interstitial lung disease, Smoking-related disease, Idiopathic interstitial pneumonia

\section{Disease name and synonyms}

Respiratory bronchiolitis-associated interstitial lung disease (RB-ILD, ORPHA79127).

The disease has not been referred to by any other synonym in medical literature.

\section{Definition}

Respiratory bronchiolitis-associated interstitial lung disease (RB-ILD) is a rare, mild inflammatory pulmonary disorder that occurs almost exclusively in current or former heavy smokers, usually between the third and sixth decades, most likely with no gender predilection. It is characterized by shortness of breath and cough, pulmonary function abnormalities of obstructive, restrictive, or mixed pattern, and high resolution computed tomography (HRCT) scans showing centrilobular micronodules, ground-glass opacities, and peribronchiolar thickening, often accompanied by tobacco-related centrilobular emphysema [1,2].

\footnotetext{
* Correspondence: asieminska@gumed.edu.pl

Department of Allergology and Pneumonology, Medical University of Gdansk, Debinki Str 7, Gdansk 80-211, Poland
}

RB-ILD is a combined respiratory bronchiolitis (RB) and interstitial lung disease (ILD). The first component of the disease - RB - was first described in 1974 as an incidental histologic lesion in the lungs of young asymptomatic cigarette smokers who died from nonpulmonary causes [3]. These pathological changes included accumulation of pigmented macrophages within the bronchioles and the peribronchiolar alveolar spaces in association with minimal chronic inflammation in the bronchiolar walls and the neighboring interstitium, and were rarely found in nonsmokers [3]. Since that initial report, RB has been recognized as an extremely common histological lesion thought to occur in virtually all cigarette smokers, and the term refers to the universal inflammatory reaction in respiratory bronchioles, known as a smoker's bronchiolitis [4-6].

Subsequently, in 1987, Myers et al. described six patients' smoking histories that had an extent of alveolar accumulation of pigmented macrophages and mural inflammation on surgical biopsy specimens that was severe enough to produce clinical, physiologic, and imaging features of ILD [6]. This clinicopathological entity was termed "respiratory 
bronchiolitis-associated interstitial lung disease" (RB-ILD). Contrary to RB alone, RB-ILD is an uncommon disorder found in a far smaller proportion of smokers $[4,6]$. Some authors have regarded RB-ILD as an exaggerated RB with more extensive peribronchiolar interstitial fibrosis [7-9], while others $[4,6]$ have considered the histologic features of $\mathrm{RB}$ and RB-ILD indistinguishable and have separated the two based on the presence of clinical evidence of ILD.

RB-ILD has been incorporated into the broad category of diffuse parenchymal lung diseases that are also known as the interstitial lung diseases (ILDs). Within this large heterogeneous group of diseases, mostly of unknown etiology, RB-ILD belongs to a subgroup termed idiopathic interstitial pneumonias (IIPs) [1,10]. The classification of IIPs was first standardized in 2002 by American Thoracic Society (ATS) and European Respiratory Society (ERS) consensus that combined the histopathologic pattern of IIPs seen on lung biopsy with clinical findings in order to establish a final clinicopathologic diagnosis $[1,11]$. According to a recent update, the ATS/ERS classification of IIPs has abstracted a group of six major IIPs, including RBILD, from rare IIPs and unclassifiable IIPs [10].

Most insight into RB-ILD to date has been provided by five case series $[6-8,12,13]$ totaling 78 patients with biopsy-proven RB-ILD, These studies have provided descriptions of clinical and physiological features, pathological and radiographic/HRCT appearances, and outcomes of the disease. Additionally, there have been several other reports focused mainly on the differential diagnosis of RB-ILD based on radiological findings [9] and their correlation with clinical and physiological findings [14], or on follow-up after smoking cessation [14,15].

In spite of the substantial clinical heterogeneity of these studies, it has been consistently reported that patients with RB-ILD are usually ever cigarette smokers with at least 30 pack-years at the time of diagnosis $[6-9,12,13]$. Only incidental single cases of RB-ILD in non-smokers have been reported, including patients exposed to second-hand smoke $[8,16]$. The age at symptom onset ranges from 22 to 70 years, with median values of $36-54$ years $[6-8,12,13]$. When the disease occurred at a younger age, it was usually in heavy smokers who had smoked 2 to 3 packs of cigarettes for at least 10 years. However, the issue of gender predilection remains unclear. Some reports have indicated that men were more often affected than women $[6,9,12]$, while others have reported contradictory data [13] or no gender predilection [8]. Therefore, the viewpoint that there is no gender predilection seems most likely to be accurate [2].

\section{Epidemiology}

The prevalence and incidence of RB-ILD is unknown and has remained difficult to assess for many years. Available epidemiological data on RB-ILD are sparse, and eventually represent a calculation of relative frequency of RB-ILD among ILDs, or more specifically, among IIP cases.

In several earlier case series, the incidence of RB-ILD was usually estimated together with that of desquamative interstitial pneumonia (DIP), and these two IIPs were found to account for $10 \%-17 \%$ of the study samples [17-20]. RB-ILD was recorded separately in only two of these case series, and accounted for $2 \%$ and $13 \%$ of cases $[17,20]$.

Several more recent studies have provided additional data on the epidemiology of ILDs under the current ATS/ERS consensus on the classification of IIPs and have even calculated the relative frequency of RB-ILD among them [21-25]. A wide epidemiological study conducted in Greece found that the prevalence and incidence of RB-ILD were 0.07 per 100,000 and 0.04 per 100,000 [23], respectively.

In contrast, in the Saudi Arabian register of newly diagnosed ILD cases, RB-ILD cases accounted for $5.5 \%$ of all types of IIPs [24]. A German pathology review of ILD cases revealed 9.5\% of RB-ILD cases among all types of IIPs [25].

\section{Clinical description}

History and physical

The onset of RB-ILD is usually insidious with exertional dyspnea, symptomatic wheezing, and persistent cough, which may be non-productive, developing over a course of weeks or months $[6-8,13]$. This clinical picture can be masked by concomitant smoking-related chronic bronchitis. RB-ILD may also be diagnosed in asymptomatic patients with functional impairment and chest radiograph or HRCT abnormalities. In most cases, RB-ILD is not a disabling disease and patients display only mild symptoms. However, some patients may have significant dyspnea and hypoxemia because of more extensive ILD [1]. Chest pain and weight loss were less frequent and hemoptysis and fever were reported incidentally [8], and might have resulted from underlying disease including lower respiratory tract infection [26]. In the literature, there is only one case of an acute presentation of RB-ILD with fevers and sweats as general symptoms, and increasing breathlessness and non-productive cough as respiratory symptoms [27].

On physical examination, although not universal, bibasilar end-inspiratory crackles are the most common signs. A case series report by Portnoy et al. [13], that included a sample size that was twice that of the next largest sample and represented the single largest experience reported to date, showed wheezing as a common sign in $69 \%$ of RBILD cases, significantly more frequent than in prior reports $[4,7,8]$.

Digital clubbing was either not reported at all $[6,7]$ or was an uncommon sign with an incidence not exceeding $25 \%[8,12,13]$. A single reported case of digital clubbing in RB-ILD probably caused by lung cancer that was detected at follow up [28] might warrant that occurrence of clubbing in an RB-ILD patient with a typical average of a 30-40-year 
smoking history should cause the clinician to suspect an occult tumor [6].

\section{Physiologic changes}

Pulmonary function tests in patients with minimal symptoms usually reveal a mild to moderate decrease in DLco. In patients with more severe symptoms, both airway obstruction and restriction or occasionally an isolated increase in residual volume may be found [6]. These features result from the variable extent of both RB-ILD and centrilobular emphysema in different cases. Patients with more advanced clinical manifestations of disease may have more significant reductions in carbon monoxide transfer [29].

The large case series reported by Portnoy et al. showed that an obstructive pattern was the most common pulmonary function defect (47\%), while pure restriction or mixed defects were less common ( $31 \%$ and $9 \%$, respectively), and that $13 \%$ of patients had normal spirometry data [13]. The authors of this study did not support the common reduction in DLco in patients with RB-ILD. Normal initial DLco values were demonstrated in $36 \%$ of patients, suggesting lower sensitivity of that marker of clinically significant disease than had been previously indicated [6-8,12].

\section{Natural history and prognosis}

Definitive reports of natural history of RB-ILD are not available due to the relatively small number of patients studied to date $[6-8,12,13]$. A few earlier case series showed that most patients with RB-ILD had a relatively stable clinical course [12]. Subsequently, Portnoy et al's case series on the long-term outcomes of RB-ILD did not support a benign course of RB-ILD, although prolonged survival was still common [13]. Symptomatic and physiologic improvements were relatively rare, and neither abstaining from cigarettes nor pharmacologic intervention regularly led to clinically significant benefit. In turn, regardless of smoking status, corticosteroids, or other therapies, self-reported subjective deterioration in overall status or of specific symptoms was commonly observed, as were objective signs of clinical worsening determined by spirometry and gas exchange measurements. Symptoms and physiology improved in only a minority of patients, although the study did agree that prolonged survival could be expected in most patients with RB-ILD and that mortality secondary to progressive ILD is rare.

\section{Etiology and pathogenesis}

RB-ILD corresponds to changes within pulmonary interstitium that are known to occur almost exclusively in current heavy cigarette smokers. However, the pathologic mechanisms that lead to these changes remain unclear, and RB-ILD is regarded as a manifestation of lung parenchymal response to inhaled cigarette smoke in both animals and humans [30,31]. The key feature of this manifestation is the bronchiolocentric accumulation of yellow-brown pigmented macrophages and cellular or fibrotic inflammation with associated clinical features of ILD $[6,8,14]$. The pigment in these so called "smokers' macrophages" represents cigarette smoke constituents, most notably kaolinite (aluminum silicate) [32].

RB-ILD is commonly viewed as an amplified respiratory bronchiolitic response that, compared to the solely bronchiole-centered lesions seen in RB, leads additionally to interstitial and air space inflammation and fibrosis extending to the nearby alveoli.

At the same time, RB-ILD has been distinguished from the more exaggerated panlobular diffuse mild-to-moderate interstitial fibrosis and massive accumulation of macrophages in the alveoli described in DIP [33]. Both of these uncommon conditions have significant clinical and histopathologic overlap [34] and were suggested to be synonymous $[7,11,35]$. Together with a common pattern of pathological abnormalities, RB-ILD and DIP show radiological overlap. The only features that may differentiate these two IIPs are the distribution and the extent of the lesions. This observation may again raise the question of whether RB-ILD and DIP represent different points along the spectrum of the same disease $[1,9,36]$, although $R B$, RB-ILD, and DIP representing various degrees of severity of the same process caused by long-time tobacco smoking is not commonly considered.

Because of the evidence of the etiological association with cigarette smoking, the latest update of the ATS/ERS classification of IIPs has grouped RB-ILD and DIP together within the major IIPs as smoking-related lung disease [10]. However, the pathogenic mechanism that links tobacco-smoke exposure to these entities has not yet been elucidated [37].

Although originally postulated by Niewoehner that RB alone could be the precursor of centriacinar emphysema [3], it remains largely unclear whether RB-ILD progresses to emphysema, despite a few studies suggesting such a possibility [38,39].

\section{Diagnosis}

It is increasingly accepted that a diagnosis of RB-ILD is secure when based upon typical HRCT findings (groundglass opacities and centrilobular nodules) in a current smoker, especially when bronchoalveolar lavage (BAL) findings (the presence of smokers' macrophages and the absence of lymphocytosis) are also compatible. In case of the assumption of an ILD other than RB-ILD, a surgical biopsy may be necessary $[1,10]$.

\section{Diagnostic methods and criteria} Histologic features of surgical biopsy specimens According to the international consensus classification of the IIPs, the histopathologic diagnosis of RB-ILD 
requires the presence of brownish pigmented macrophages within respiratory bronchioles and alveolar ducts, associated with a patchy submucosal and peribronchiolar chronic infiltrate of lymphocytes and histiocytes (Figure 1). The common findings also include mild bronchiolar and peribronchiolar alveolar fibrosis that stretches contiguous alveolar septa and leads to architectural distortion as well as centrilobular emphysema [1].

\section{Findings of bronchoalveolar lavage and transbronchial biopsy}

BAL findings in RB-ILD patients usually do not differ from those seen in otherwise normal healthy smokers and include an increased total number of cells with a normal cellular differential analysis [13] or an increase in the percentage of macrophages [15]. These alveolar macrophages contain brownish, golden, or black tobacco pigment inclusions In the absence of these cells, an alternative diagnosis should be considered. A modest increase in neutrophils may also be present [40].

Transbronchial biopsy might be useful in distinguishing RB-ILD from hypersensitivity pneumonitis (HP) or sarcoidosis, but not from DIP. In one case, transbronchial biopsy was sufficient to establish a diagnosis of acute RB-ILD [27].

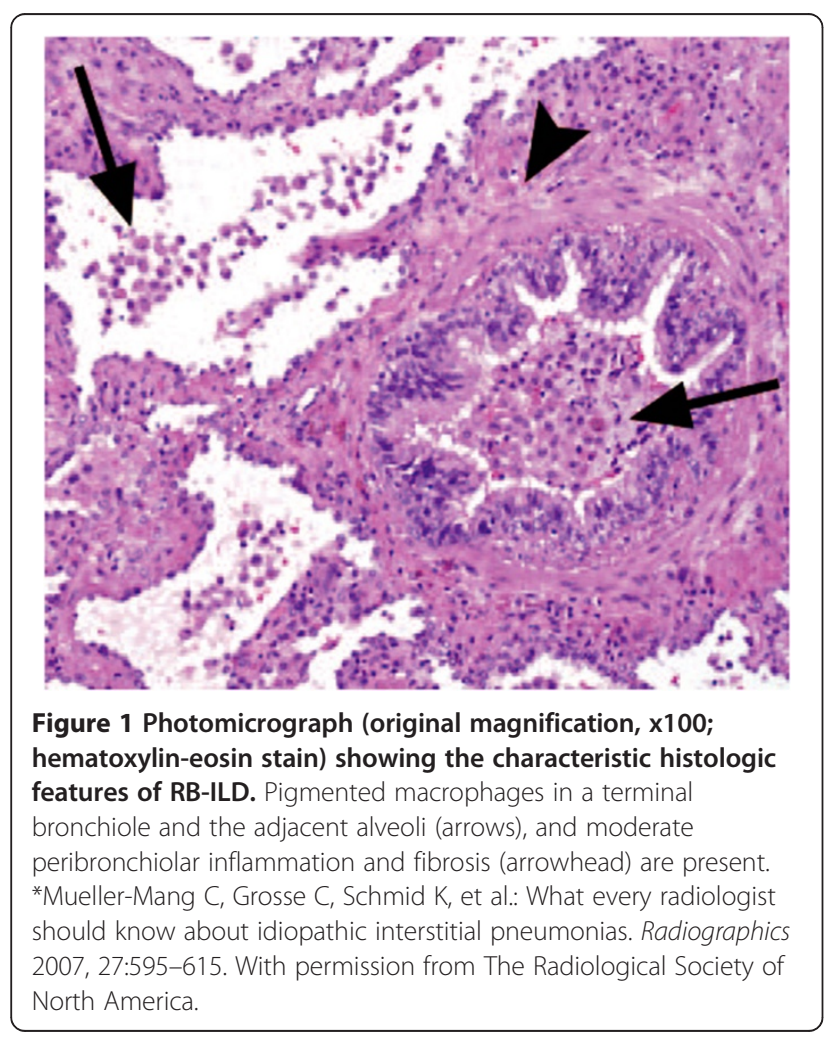

\section{Radiographic findings}

Radiographic findings are usually relatively subtle $[6,7,9,14]$. Normal chest radiographs have been reported in $20 \%$ to $28 \%$ of patients with histologically-proven RBILD $[6,7,41]$, and in a single case, both normal chest $x$-ray and HRCT appearance were reported [41]. When present, typical chest $\mathrm{x}$-ray findings in patients with RB-ILD may include fine reticulonodular interstitial opacities, which are diffuse [7] or are predominant in basal lung areas [6]. The chest radiograph may also reveal signs of thickening of the walls of the central and peripheral bronchi $[6,7,9]$.

HRCT findings in RB-ILD patient are shown in Figure 2. Some differences exist between studies in terms of frequency of particular changes and their zonal predominance at HRCT. The most frequent findings reported by Park et al. were central and peripheral bronchial wall thickening (in $90 \%$ and $86 \%$ of patients, respectively) and centrilobular nodules (71\%) [14]. Ground-glass opacity was observed significantly less frequently than in the earlier series (67\% vs. $100 \%$ of patients) [12]. Although in a few other studies, the upper lung zones were mostly affected $[8,9,14]$, Park et al. did not report any zonal predominance. Centrilobular emphysema in the upper lobes was reported consistently as a common (50\%-57\%) but not severe finding $[8,9,14]$. Patchy areas of hypoattenuation were less common (38\%) and reported to have lower lung predominance [14]. Most probably, this hypoattenuation reflects air trapping due to small airway disease [14,42]. However, a tree-in-bud pattern, which is a common manifestation of small-airway-disease, was rarely reported [43]. The frequency of interstitial fibrosis in RB-ILD patients, as determined by the presence of intralobular lines and a

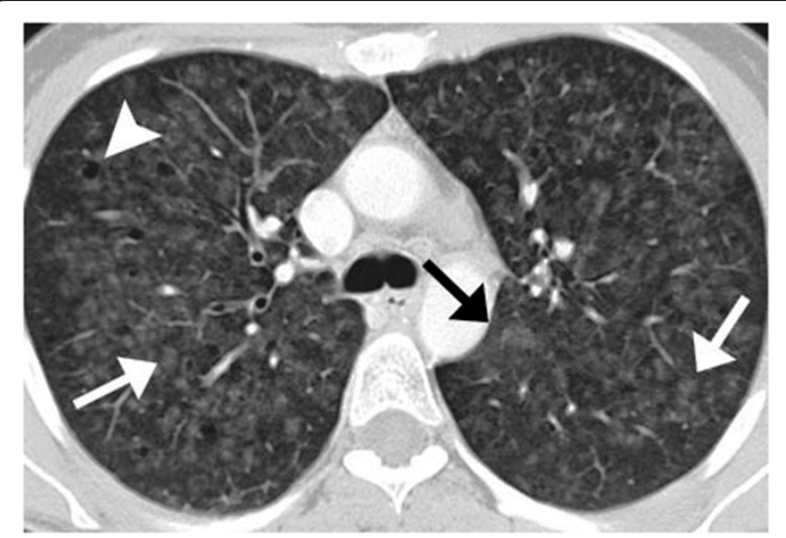

Figure 2 RB-ILD in a 44-year-old woman with a 20 pack-year smoking history. HRCT image of the upper lung lobes shows centrilobular nodules (white arrows), patchy ground-glass opacities (black arrow), and mild coexisting centrilobular emphysema (arrowhead). *Mueller-Mang C, Grosse C, Schmid K, et al.: What every radiologist should know about idiopathic interstitial pneumonias. Radiographics 2007, 27:595-615. With permission from The Radiological Society of North America. 
reticular pattern, differed significantly in various studies, and ranged from $20 \%$ to $75 \%[8,9,13,41]$, while honeycombing was less common $(0 \%-12 \%)[8,12,13]$.

\section{Pulmonary function tests}

Mixed, predominantly obstructive abnormalities, often combined with hyperinflation and usually associated with a mild to moderate reduction in DLco are the most frequently reported findings in patients diagnosed with RB-ILD $[6-8,13]$. Airflow obstruction is usually mild.

\section{Differential diagnosis}

First, the entities that share a common pathogenesis and clinical-histopathological pattern with RB-ILD, i.e., RB and DIP, should be taken into account in the differential diagnosis. However, separating RB and RB-ILD by sole histopathological criteria remains controversial; moreover, the two entities can also rarely be distinguished solely by the nature and extent of HRCT findings, because there is no arbitrary cut-off point for which the extent of disease at HRCT evolves from RB to RB-ILD [5-9]. Therefore, it has been proposed that the distinction between RB and RB-ILD should be based not on histological or radiological appearance alone, but on all markers of disease severity taken together, including clinical symptoms, pulmonary function tests results, and HRCT findings. On the contrary, lung biopsy is sometimes required to distinguish RB-ILD from DIP. Although RB-ILD can usually be distinguished from DIP radiologically with $\mathrm{HRCT}$, there is considerable overlap between these two IIPs. In the face of the different prognoses associated with these entities [7], correct diagnosis is of considerable importance. For the pathologist, a challenge in the differential diagnosis might be the separation of the fibrotic form of RB-ILD that was suggested by Yousem from DIP [44]. This histologic appearance has been linked to a macrophage-poor variant of DIP, and the term RB-ILD with fibrosis has been suggested to represent a subset of diagnoses at the extreme end of the RB-ILD spectrum [44]. Further, both RB-ILD and DIP need to be differentiated from airspace enlargement with fibrosis (AEF), the changes which have been incidentally reported on histologic and HRCT examination in smokers, and in comparison to emphysema marked by more extensive interstitial fibrosis $[45,46]$. In addition, the histopathological distinction between RB-ILD and centrilobular emphysema, which is usually associated with RB and sometimes with interstitial fibrosis, appears to be another challenge. It has been postulated that functional abnormalities might help in making a definite diagnosis [47].

Next, other ILDs, including nonspecific interstitial pneumonia (NSIP) and subacute HP, should be taken into account in differential diagnosis. Usually these can be effectively excluded based on the HRCT findings: the ground-glass opacity observed in RB-ILD is usually patchier than that seen in NSIP or acute or subacute HP; additionally, a greater extent of micronodules in acute or subacute HP and the presence of diffuse bronchial wall thickening in RB-ILD may help in differentiating the two entities [14]. Additionally, a history of smoking (rare in HP) together with BAL findings (lymphocytosis in HP) can assist in differentiating RB-ILD from subacute HP $[14,15,48]$.

\section{Management and disease course}

The indications for treatment of RB-ILD are often marginal. Although to date, the regression of findings consistent with RB-ILD after discontinuation of smoking not accompanied by corticosteroid therapy has been reported in few patients $[6,15,49]$, smoking cessation is considered the most important factor in the management of RB-ILD. Because the majority of patients in reported case series received corticosteroid and/or immunosuppressive therapy along with smoking cessation, it is not yet completely understood whether smoking cessation alone can improve outcomes in RB-ILD $[7,8,12,14,49]$. It is also unclear whether patients with RB-ILD benefit from corticosteroid therapy with regard to the natural history of the disease. In one report, at follow-up HRCT after corticosteroid treatment and smoking cessation, the extent of bronchial wall thickening, centrilobular nodules, and ground-glass opacities had decreased in $43 \%$ of patients, while areas of hypoattenuation had increased as emphysema was irreversible on follow up [14]. However, other studies have presented cases with no change or deterioration despite corticosteroid therapy $[8,12]$, or the failure of corticosteroid tapering and discontinuation even in the absence of smoking [12].

So far, the prognosis for patients with RB-ILD has been good, with prolonged survival expected in most cases. Until now only one death from progressive lung disease during a median follow-up period of 7 years has been reported during a study that explored the outcomes in 32 RB-ILD patients [13]. However, in the absence of other longitudinal data, the median survival time can be only approximate. As was suggested in the study just mentioned, at least $75 \%$ of the patients would be expected to live at least 7 years [13], although this case series with long follow-up did not support the contention that RB-ILD is a benign disease. Clinical worsening was common, as well as worsening of spirometry results and gas exchange, regardless of smoking status and treatment ordered, including corticosteroids [13].

To summarize, the course of RB-ILD is heterogeneous, often with no functional improvement and with disease progression despite smoking cessation and treatment $[8,12,13]$. In contrast to previous assumptions, RB-ILD is now less commonly regarded as a benign entity. 


\section{Unresolved questions}

1. What are the incidence and prevalence of the RB-ILD?

2. What is the relationship of RB-ILD to other forms of IIPs?

3. What is the relationship of RB-ILD to DIP, and does RB-ILD progress to DIP?

4. What are the potential causes of RB-ILD other than cigarette smoking?

5. Does RB-ILD advance to emphysema?

6. Do smoking cessation and treatment with corticosteroids alter the natural history of RB-ILD?

7. Do any HRCT features of RB-ILD improve following smoking cessation?

\section{Conclusions}

RB-ILD is a rare and not fully understood disease. This clinicopathologic syndrome combines RB and ILD. Because of the evidence of the etiological association with cigarette smoking, RB-ILD is referred to as smokingrelated ILD. However, the pathogenic mechanism that links tobacco-smoke exposure to this entity has not yet been clarified, and thus it is still regarded as idiopathic disease. RB-ILD has significant pathological/clinical/radiological overlap with DIP, what raises the question whether RB-ILD and DIP represent different points along the spectrum of the same disease. Further clarification of the relationship between RB-ILD and DIP is warranted. The exact prevalence and incidence of RB-ILD is unknown. Similarly, long-term outcome of RB-ILD awaits recognition. The condition seems not to be as benign as it was previously considered. Some patients respond favorably to corticosteroids and/or smoking cessation, but often the disease progresses despite smoking cessation and treatment. It also remains unclear whether RB-ILD progresses to emphysema.

Competing interests

Both authors declare that they have no competing interests.

\section{Authors' contributions}

$\mathrm{AS}$ and KK participated in the process of the literature review and in the drafting the final manuscript. In addition, AS supervised the project. Both authors read and approved the final manuscript.

\section{Acknowledgement}

We would like to thank Krzysztof Specjalski MD from Allergology Department of Medical University of Gdansk, for initial proofreading the manuscript. Authors thank Editage for providing editorial assistance for this article This work was supported by university grant ST-553.

Received: 7 May 2014 Accepted: 27 June 2014

Published: 11 July 2014

\section{References}

1. Travis WD, King TE Jr, Bateman ED, Lynch DA, Capron F, Center D, Colby TV, Cordier JF, duBois RM, Galvin J, Grenier P, Hansell DM, Hunninghake G, Kitaichi M, Muller NL, Myers J, Nagai S, Nicholson A, Raghu G, Wallaert B: American thoracic society/European respiratory society international multidisciplinary consensus classification of the idiopathic interstitial pneumonias. Am J Respir Crit Care Med 2002, 165:277-304. co-chairs.
2. Wells $A U$, Nicholson AG, Hansell DM: Challenges in pulmonary fibrosis 4: smoking-induced diffuse interstitial lung diseases. Thorax 2007, 62:904-10.

3. Niewoehner DE, Kleinerman J, Rice DB: Pathologic changes in the peripheral airways of young cigarette smokers. N Engl J Med 1974, 291:755-88.

4. Fraig M, Shreesha U, Savici D, Katzenstein AL: Respiratory bronchiolitis: a clinicopathologic study in current smokers, ex-smokers, and neversmokers. Am J Surg Pathol 2002, 26:647-53.

5. Cosio M, Ghezzo H, Hogg JC, Corbin R, Loveland M, Dosman J, Macklem PT: The relations between structural changes in small airways and pulmonary function tests. N Engl J Med 1978, 298:1277-81.

6. Myers $J \mathrm{~L}$, Veal CF Jr, Shin MS, Katzenstein AL: Respiratory bronchiolitis causing interstitial lung disease: a clinicopathological study of six cases. Am Rev Respir Dis 1987, 135:880-4.

7. Yousem SA, Colby TV, Gaensler EA: Respiratory bronchiolitis-associated interstitial lung disease and its relationship to desquamative interstitial pneumonia. Mayo Clin Proc 1989, 64:1373-80.

8. Moon J, du Bois RM, Colby TV, Hansell DM, Nicholson AG: Clinical significance of respiratory bronchiolitis on open lung biopsy and its relationship to smoking related interstitial lung disease. Thorax 1999, 54:1009-14.

9. Heyneman LE, Ward S, Lynch DA, Remy-Jardin M, Jonkoh T, Müller NL: Respiratory bronchiolitis, respiratory bronchiolitis-associated interstitial lung disease, and desquamative interstitial pneumonia: different entities or part of the spectrum of the same disease process. Am J Roentgenol 1999, 173:1617-22.

10. Travis WD, Costabel U, Hansell DM, King TE Jr, Lynch DA, Nicholson AG, Ryerson CJ, Ryu JH, Selman S, Wells AU, Behr J, Bouros D, Brown KK, Colby TV, Collard HR, Cordeiro CR, Cottin V, Crestani B, Drent M, Dudden RF, Egan J, Flaherty K, Hogaboam C, Inoue Y, Johkoh T, Kim DS, Kitaichi M, Loyd J, Martinez FJ, Jeffrey Myers J, et al: An official American thoracic society/ European respiratory society statement: update of the international multidisciplinary classification of the idiopathic interstitial pneumonias. Am J Respir Crit Care Med 2013, 188:733-748.

11. Katzenstein AA, Myers $J$ : Idiopathic pulmonary fibrosis: clinical relevance of pathologic classification. Am J Respir Crit Care Med 1998, 157:1301-15.

12. Ryu JH, Myers JL, Capizzi SA, Douglas WW, Vassallo R, Decker PA: Desquamative interstitial pneumonia and respiratory bronchiolitisassociated interstitial lung disease. Chest 2005, 127:178-184.

13. Portnoy J, Veraldi KL, Schwarz MI, Cool CD, Curran-Everett D, Cherniack RM, King TE Jr, Brown KK: Respiratory bronchiolitis-interstitial lung disease: long-term outcome. Chest 2007, 131:664-671.

14. Park JS, Brown KK, Tuder RM, Hale VA, King TE Jr, Lynch DA: Respiratory bronchiolitis-associated interstitial lung disease: radiologic features with clinical and pathologic correlation. J Comput Assist Tomogr 2002, 26:13-20.

15. Nakanishi M, Demura Y, Mizuno S, Ameshima S, Chiba Y, Miyamori I, Itoh $\mathrm{H}_{\text {, }}$ Kitaichi M, Ishizaki T: Changes in HRCT findings in patients with respiratory bronchiolitis-associated interstitial lung disease after smoking cessation. Eur Respir J 2007, 29:453-461.

16. Woo OH, Yong HS, Oh Y, Lee SY, Kim HK, Kang E: Respiratory bronchiolitisassociated interstitial lung disease in a nonsmoker: radiologic and pathologic findings. AJR 2007, 188:412-414.

17. Bjoraker JA, Ryu JH, Edwin MK, Myers JL, Tazelaar HD, Schroeder DR, Offord KP: Prognostic significance of histopathologic subsets in idiopathic pulmonary fibrosis. Am J Respir Crit Care Med 1998, 157:199-203.

18. Nicholson AG, Colby TV, Dubois RM, Hansell DM, Wells AU: The prognostic significance of the histologic pattern of interstitial pneumonia in patients presenting with the clinical entity of cryptogenic fibrosing alveolitis. Am J Respir Crit Care Med 2000, 162:2213-2217.

19. Travis WD, Matsui K, Moss J, Ferrans VJ: Idiopathic nonspecific interstitial pneumonia: prognostic significance of cellular and fibrosing patterns: survival comparison with usual interstitial pneumonia and desquamative interstitial pneumonia. Am J Surg Pathol 2000, 24:19-33.

20. Flaherty KR, Toews GB, Travis WD, Colby TV, Kazerooni EA, Gross BH, Jain A, Strawderman RL 3rd, Paine R, Flint A, Lynch JP 3rd, Martinez FJ: Clinical significance of histological classification of idiopathic interstitial pneumonia. Eur Respir J 2002, 19:275-83.

21. Tinelli C, De Silvestri A, Richeldi L, Oggionni T: The Italian register for diffuse infiltrative lung disorders (RIPID): a four-year report. Sarcoidosis Vasc Diffuse Lung Dis 2005, 22:4-8.

22. Xaubet A, Ancochea J, Morell F, Rodriquez-Arias JM, Villena V, Blanquer R, Montero C, Sueiro A, Disdier C, Vendrell M, Spanish Group on Interstitial 
Lung Diseases, SEPAR: Report on the incidence of interstitial lung diseases in Spain. Sarcoidosis Vasc Diffuse Lung Dis 2004, 21:64-70.

23. Karakatsani A, Papakosta D, Rapti A, Antoniou KM, Dimadi M, Markopoulou A, Latsi P, Polychronopoulos V, Birba G, Ch L, Bouros D, Hellenic Interstitial Lung Diseases Group: Epidemiology of interstitial lung diseases in Greece. Respir Med 2009, 103:1122-9.

24. Alhamad EH: Interstitial lung diseases in Saudi Arabia: a single-center study. Ann Thorac Med 2013, 8:33-7.

25. Theegarten D, Müller HM, Bonella F, Wohlschlaeger J, Costabel U: Diagnostic approach to interstitial pneumonias in a single centre: report on 88 cases. Diagn Pathol 2012, 7:160-171.

26. McWilliams AM, Lake FR: Respiratory bronchiolitis associated interstitial lung disease (RB-ILD) presenting with haemoptysis. Respirology 2000 5:385-387.

27. Mavridou $D$, Laws $D$ : Respiratory bronchioloitis associated interstitial lung disease (RB-ILD): a case of an acute presentation. Thorax 2004, 59:910-911.

28. Scheidl S, Kovacs G, Stacher E, Popper H, Olschewski H: A 55-year-old craftsman with dyspnea and clubbing: a case report. Cases J 2009, 2:8579.

29. King TE Jr: Respiratory bronchiolitis-associated interstitial lung disease. Clin Chest Med 1993, 14:693-698.

30. Hammond EC, Auerbach O, Kirman D, Garfinkel L: Effects of cigarette smoking on dogs. Arch Environ Health 1970, 21:740-753.

31. Weiss W: Cigarette smoking and diffuse pulmonary fibrosis. Am Rev Respir Dis 1969, 99:67-72.

32. Brody AR, Craighead JE: Cytoplasmic inclusions in pulmonary macrophages of cigarette smokers. Lab Invest 1975, 32:125-32.

33. Liebow AA, Steer A, Billingsley JG: Desquamative interstitial pneumonia. Am J Med 1965, 39:369-404.

34. Colby TV: Bronchiolitis: pathologic considerations. Am J Clin Pathol 1998, 109:101-109.

35. Myers JL: Respiratory bronchiolitis with interstitial lung disease. Semin Respir Med 1992, 13:134-139.

36. Craig PJ, Wells AU, Doffman S, Rassi D, Colby TV, Hansell DM, du Bois RM, Nicholson AG: Desquamative interstitial pneumonia, respiratory bronchiolitis and their relationship to smoking. Histopathology 2004, 45:275-282.

37. Selman M: The spectrum of smoking-related interstitial lung disorders: the never-ending story of smoke and disease. Chest 2003, 124:1185-1187.

38. Remy-Jardin M, Edme J-L, Boulenguez C, Remy J, Mastora I, Sobaszek A: Longitudinal follow-up study of smoker's lung with thin-section CT in correlation with pulmonary function tests. Radiology 2002, 222:261-70.

39. Davies $G$, Wells $A U$, du Bois RM: Respiratory bronchiolitis associated with interstitial lung disease and desquamative pneumonia. Clin Chest Med 2004, 25:717-726

40. Hunninghake GW, Crystal RG: Cigarette smoking and lung destruction: accumulation of neutrophils in the lungs of cigarette smokers. Am Rev Respir Dis 1983, 128:833-838.

41. Holt RM, Schmidt RA, Godwin JD, Raghu G: High-resolution CT in respiratory bronchiolitis-associated interstitial lung disease. J Comput Assist Tomogr 1993, 17:46-50

42. Stern EJ, Swensen SJ, Hartman TE, Frank MS: CT mosaic pattern of lung attenuation: distinguishing different causes. AJR 1995, 165:813-816.

43. Hansell DM: Small airways diseases: detection and insights with computed tomography. Eur Respir J 2001, 17:1294-1313.

44. Yousem SA: Respiratory-bronchiolitis-asociated interstitial lung disease with fibrosis is a lesion distinct from fibrotic nonspecific interstitial pneumonia: a proposal. Mod Pathol 2006, 19:1474-1479.

45. Kawabata Y, Hoshi E, Murai K, Ikeya T, Takahashi N, Saitou Y, Kurashima K, Ubukata M, Takayanagi N, Sugita H, Kanauchi S, Colby TV: Smoking-related changes in the background lung of specimens resected for lung cancer: a semiquantitative study with correlation to postoperative course. Histopathology 2008, 53:707-714.

46. Katzenstein AL, Mukhopadhyay S, Zanardi C, Dexter E: Clinically occult interstitial fibrosis in smokers: classification and significance of a surprisingly common finding in lobectomy specimens. Hum Pathol 2010 41:316-325.
47. Churg A, Müller NL, Wright JL: Respiratory bronchiolitis/interstitial lung disease: fibrosis, pulmonary function, and evolving concepts. Arch Pathol Lab Med 2010, 134:27-32.

48. Warren C: Extrinsic allergic alveolitis: a disease commoner in nonsmokers. Thorax 1977, 32:567-573.

49. Sadikot RT, Johnson J, Loyd JE, Christman JW: Respiratory bronchiolitis associated with severe dyspnea, exertional hypoxemia, and clubbing. Chest 2000, 117:282-285.

doi:10.1186/s13023-014-0106-8

Cite this article as: Sieminska and Kuziemski: Respiratory bronchiolitisinterstitial lung disease. Orphanet Journal of Rare Diseases 2014 9:106.

\section{Submit your next manuscript to BioMed Central and take full advantage of:}

- Convenient online submission

- Thorough peer review

- No space constraints or color figure charges

- Immediate publication on acceptance

- Inclusion in PubMed, CAS, Scopus and Google Scholar

- Research which is freely available for redistribution

Submit your manuscript at www.biomedcentral.com/submit
C Biomed Central 